\title{
Analysis of Ammonium Nitrate/Urea Nitrate with Crown Ethers and Sugars as Modifiers by Electrospray Ionization-Mass Spectrometry and Ion Mobility Spectrometry
}

\author{
Chia-Wei Tsai ${ }^{1}$; Anthony Midey ${ }^{2}$; Ching $\mathrm{Wu}^{2}$; Richard A. Yost ${ }^{1 *}$ \\ ${ }^{I}$ Department of Chemistry, University of Florida, Gainesville, FL \\ ${ }^{2}$ Excellims Corporation, Acton, $M A$
}

\section{Supporting Information}

Revised and Resubmitted to Analytical Chemistry

August 24, 2016

* Corresponding Author:

Professor Richard A. Yost

Department of Chemistry

University of Florida

Gainesville, FL

Tel: (352) 392-0557

Fax: (352) 392-4651

Email: ryost@ufl.edu 


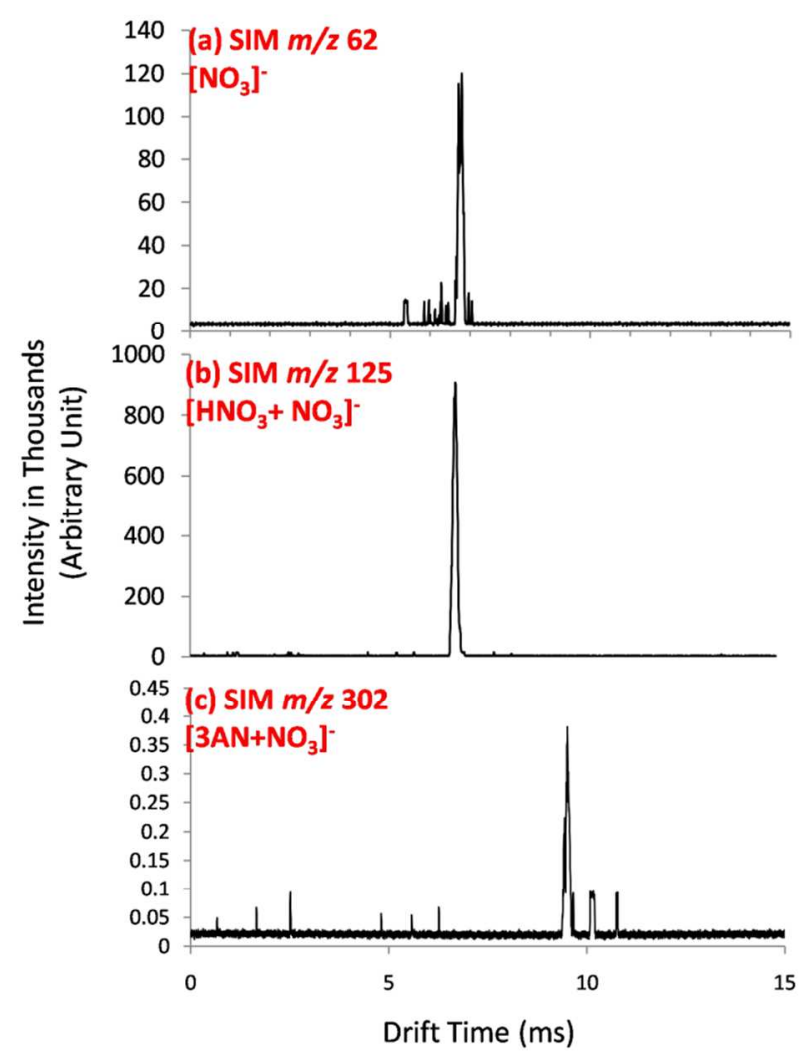

Figure S1 ESI-HPIMS ion mobility spectra from single-ion-monitoring (SIM) of AN cluster ions at $m / z 62$ and $m / z 125$ collected using MS as detector. Both $m / z 62$ and 125 were detected with 6.8 ms drift time which suggests that the nitrate ions observed originate from dissociation of the dimer. 


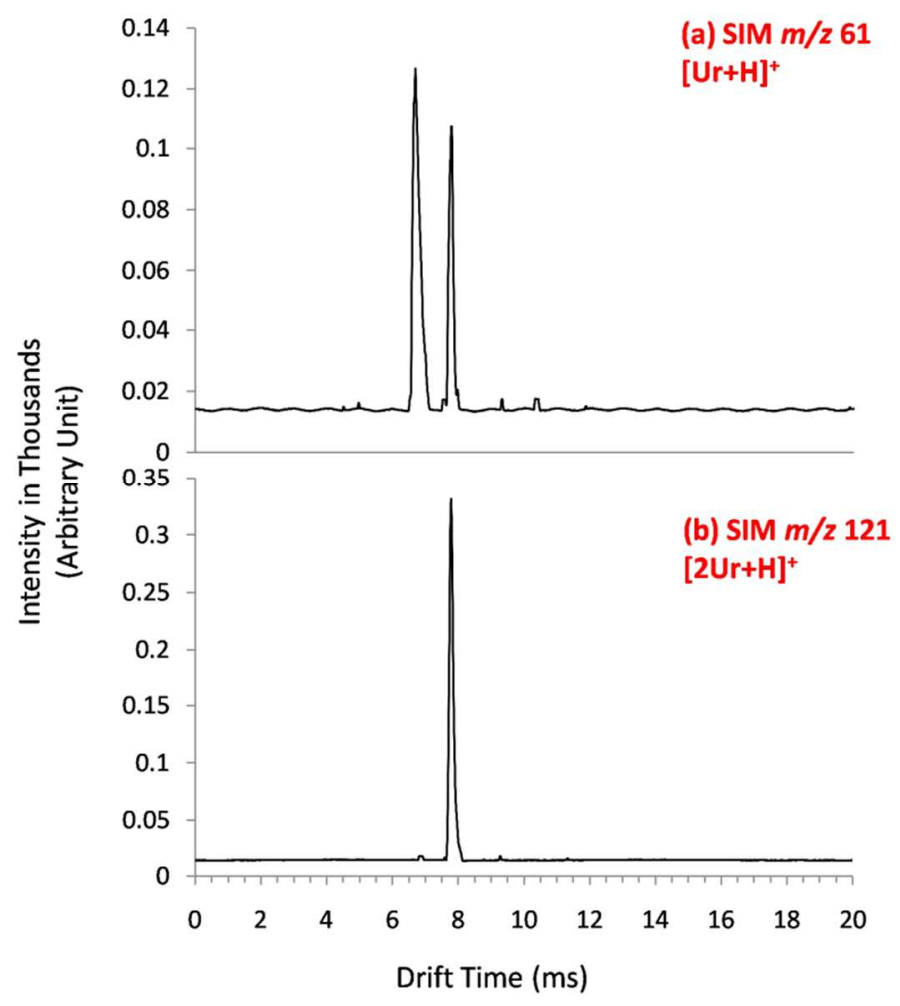

Figure S2 Positive mode ESI-HPIMS ion mobility spectra of single ion monitoring (SIM) of UN cluster ions $m / z 61$ and $m / z 121$ collected at the MS detector. 


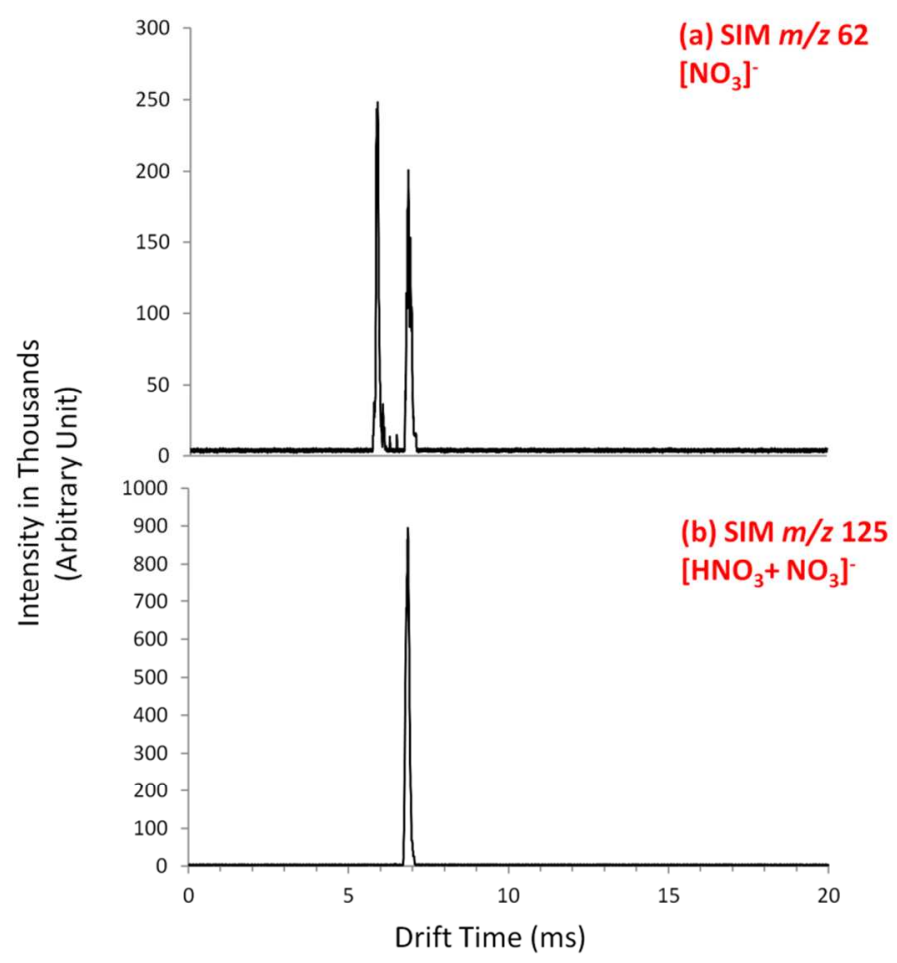

Figure S3 ESI-HPIMS ion mobility spectra using single ion monitoring with UN cluster ions in negative mode at the MS detector. 
Table S1. Relative Cavity Sizes of Crown Ethers ${ }^{26}$

\begin{tabular}{|c|c|}
\hline Polyether Ring & Cavity Size $(\AA)$ \\
\hline 12-crown-4 & $1.2-1.5$ \\
\hline 15-crown-5 & $1.7-2.2$ \\
\hline 18-crown-6 & $2.6-3.2$ \\
\hline 21-crown-7 & $3.4-4.3$ \\
\hline
\end{tabular}




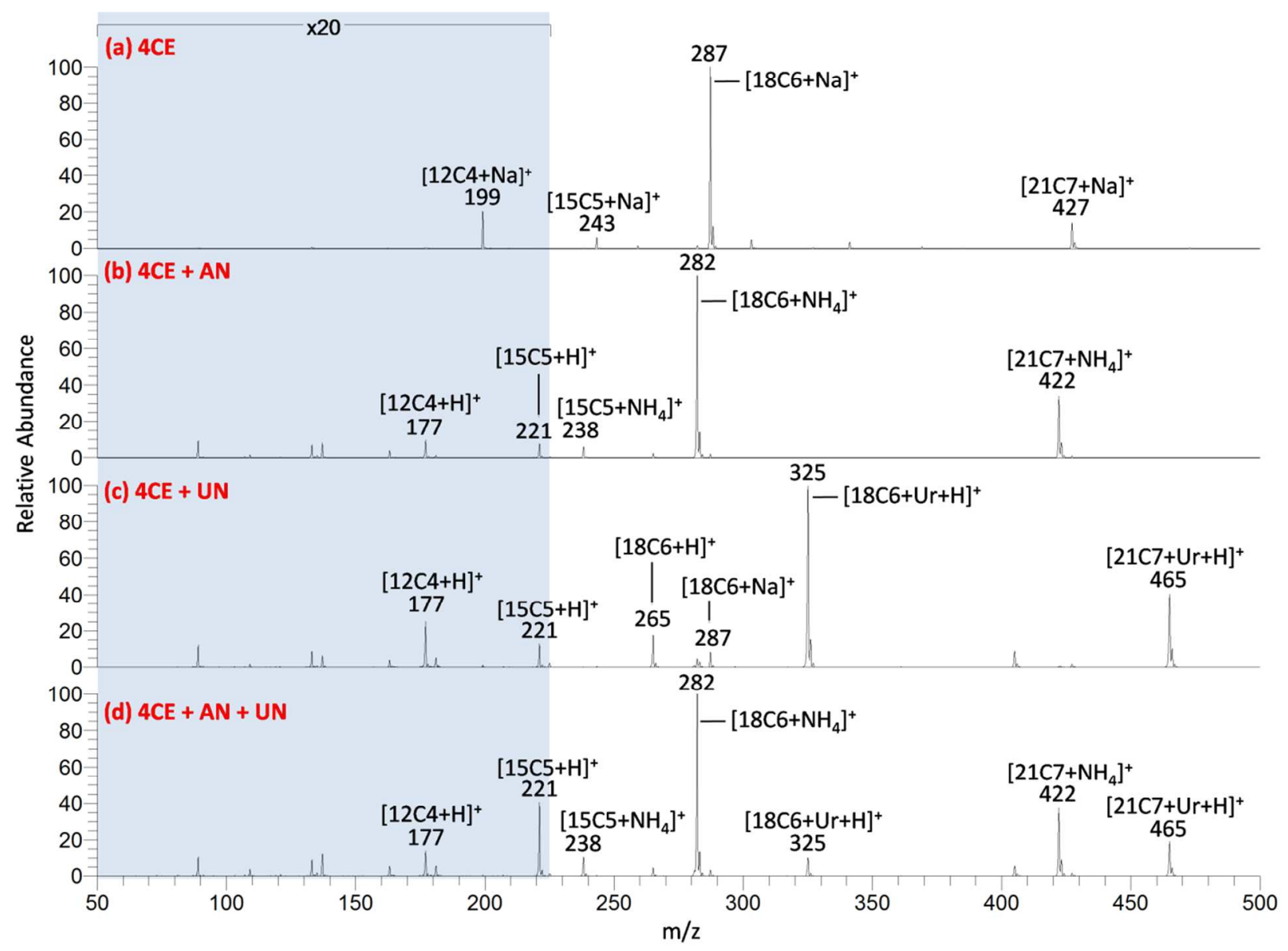

Figure S4 ESI-MS spectra of a mixture of 4 crown ethers (4CE): (a) without analyte, (b) with AN, (c) with UN, and (d) with both AN and UN. Signal intensity in the shaded area is amplified 20x. 


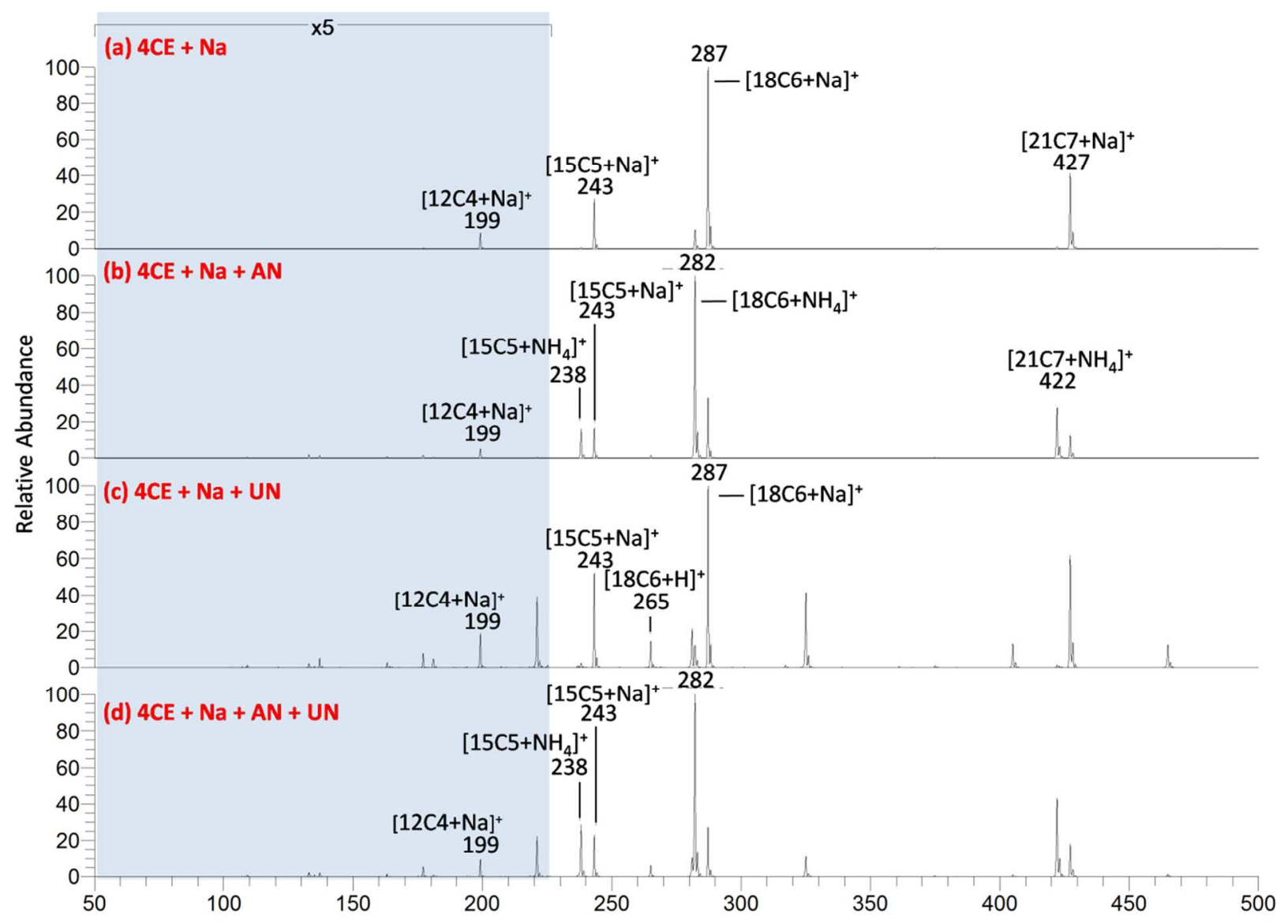

Figure S5 Study on the effects of sodium ion. ESI-MS spectra of a mixture of 4 crown ethers (4CE) with addition of sodium and (a) without analyte, (b) with AN, (c) with UN, and (d) with both AN and UN. Signal intensity in the shaded area is amplified $5 x$. 


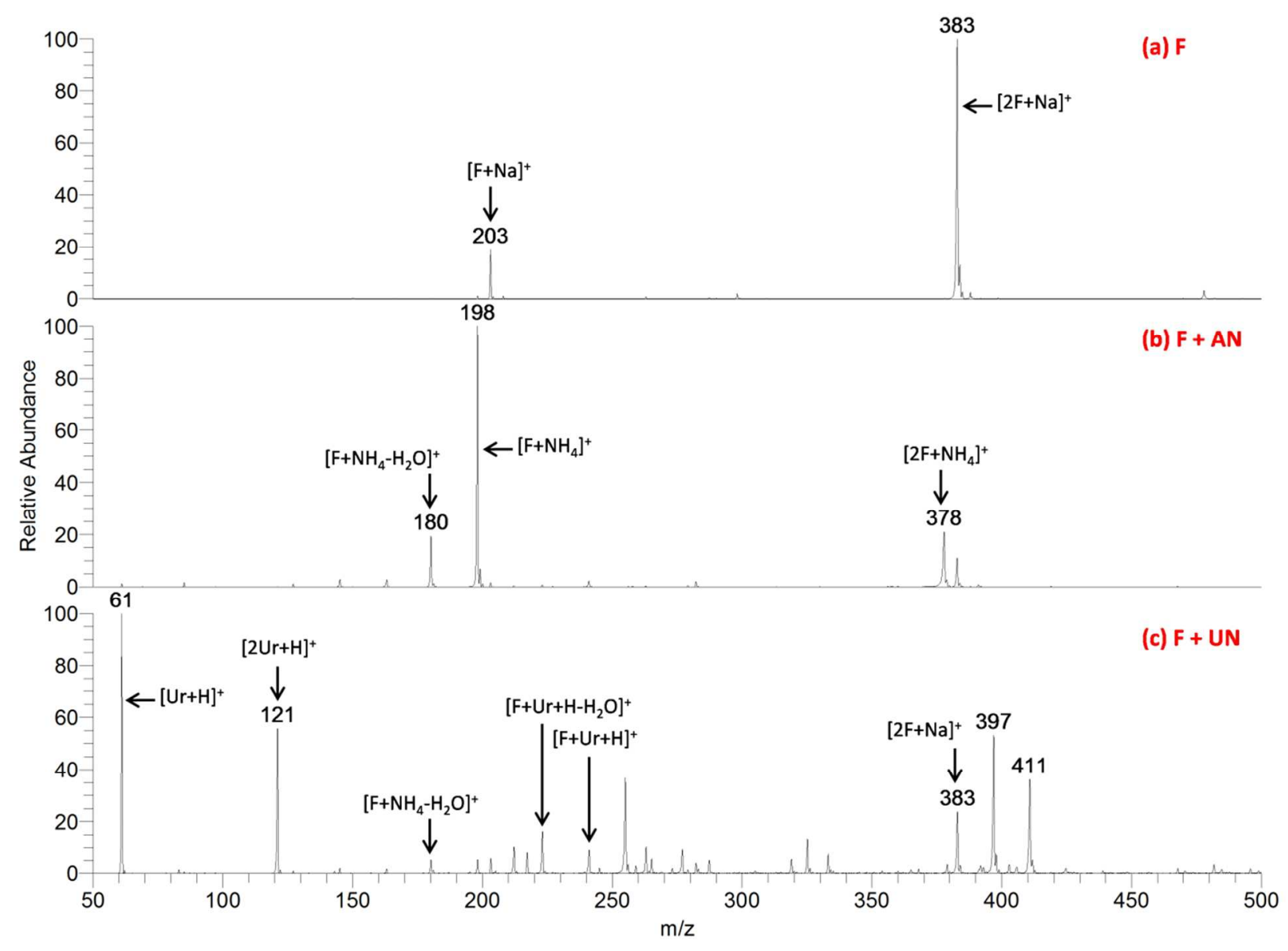

FigureS6 Positive mode ESI-MS spectra of: (a) fructose, (b) fructose with AN, and (c) fructose with UN. The results indicate that the analyte cations form adducts with fructose as well. 


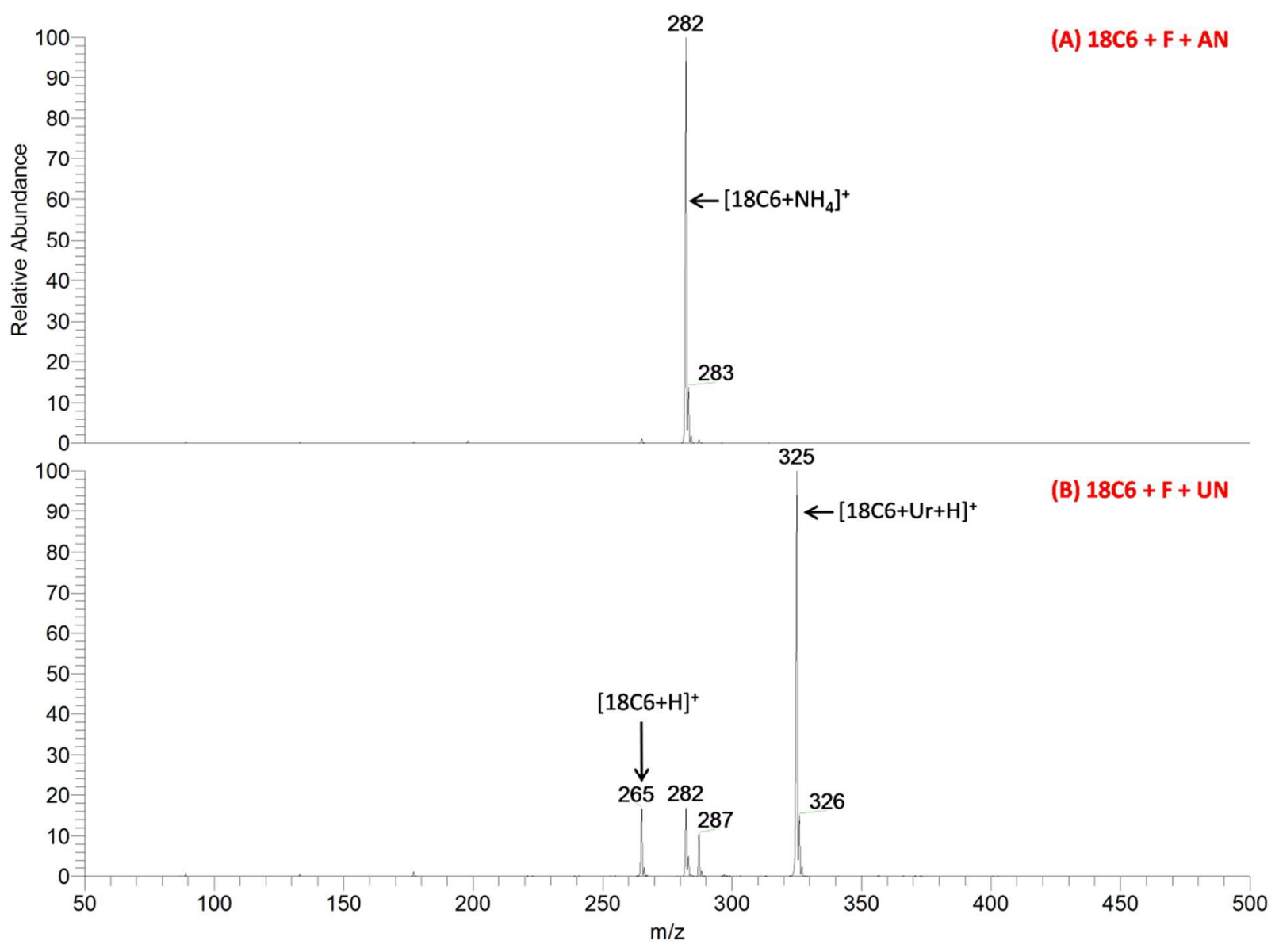

Figure S7 Positive mode ESI-MS spectra of mixtures of: (a) $18 \mathrm{C} 6$ with fructose and AN and (b) 18C6 with fructose and UN. The results indicate that 18C6 forms complexes with analyte cations more strongly than does fructose. 


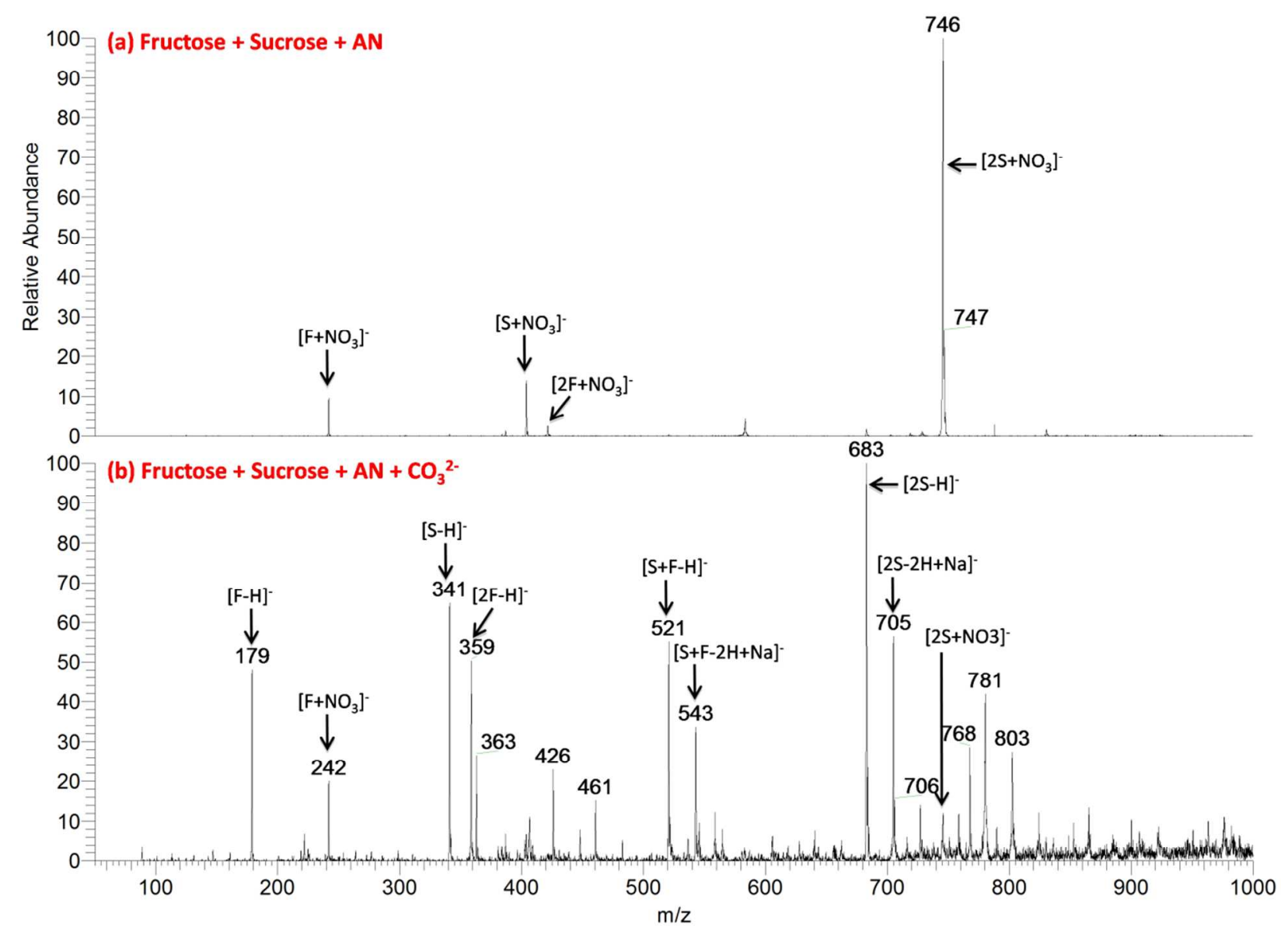

Figure S8 Negative mode ESI-MS spectra from experiments (a) comparing different sugars as modifiers and (b) comparing the effects of carbonate ion as an interferent. 